\title{
Corrigendum: Coherent assessments of Europe's marine fishes show regional divergence and megafauna loss
}

Paul G. Fernandes, Gina M. Ralph, Ana Nieto, Mariana García Criado, Paraskevas Vasilakopoulos, Christos D. Maravelias, Robin M. Cook, Riley A. Pollom, Marcelo Kovačić, David Pollard, Edward D. Farrell, Ann-Britt Florin, Beth A. Polidoro, Julia M. Lawson, Pascal Lorance, Franz Uiblein, Matthew Craig, David J. Allen, Sarah L. Fowler, Rachel H. L. Walls, Mia T. Comeros-Raynal, Michael S. Harvey, Manuel Dureuil, Manuel Biscoito, Caroline Pollock, Sophy R. McCully Phillips, Jim R. Ellis, Constantinos Papaconstantinou, Alen Soldo, Çetin Keskin, Steen Wilhelm Knudsen, Luís Gil de Sola, Fabrizio Serena, Bruce B. Collette, Kjell Nedreaas, Emilie Stump, Barry C. Russell, Silvia Garcia, Pedro Afonso, Armelle B. J. Jung, Helena Alvarez, João Delgado, Nicholas K. Dulvy and Kent E. Carpenter

Nature Ecology \& Evolution 1, 0170 (2017); published 26 May 2017; corrected 12 June 2017.

In the original version of this Article, the European Commission was mistakenly included as an affiliation for Christos D. Maravelias. His contribution to this work was exclusively completed while at the Hellenic Centre for Marine Research. All versions of the Article have been corrected. 